\title{
Stem cells and the origin of gliomas: A historical reappraisal with molecular advancements
}

\author{
Michael L Levy' \\ Allen L Hol,2 \\ Samuel Hughes ${ }^{3}$ \\ Jayant Menon' \\ Rahul Jandial ${ }^{4}$ \\ 'Division of Neurosurgery, University \\ of California, San Diego, La Jolla, \\ California, USA; ${ }^{2}$ Del E Webb \\ Neurosciences, Aging and Stem \\ Cell Research Center, The Burnham \\ Institute for Medical Research, La \\ Jolla, California, USA; ${ }^{3}$ Department \\ of Neurological Surgery, Oregon \\ Health and Science University, \\ Portland, OR, USA; ${ }^{4}$ Division \\ of Neurosurgery, Department \\ of Surgery, City of Hope Cancer \\ Center, Duarte, CA, USA
}

\begin{abstract}
The biology of both normal and tumor development clearly possesses overlapping and parallel features. Oncogenes and tumor suppressors are relevant not only in tumor biology, but also in physiological developmental regulators of growth and differentiation. Conversely, genes identified as regulators of developmental biology are relevant to tumor biology. This is particularly relevant in the context of brain tumors, where recent evidence is mounting that the origin of brain tumors, specifically gliomas, may represent dysfunctional developmental neurobiology. Neural stem cells are increasingly being investigated as the cell type that originally undergoes malignant transformation - the cell of origin - and the evidence for this is discussed.
\end{abstract}

Keywords: stem cells, gliomas, neural stem cells, brain tumors, cancer stem cells

\section{Origin of brain tumors: historical insights}

Over a century ago, the German pathologist Julius Cohnheim described the similarities between tumors and embryonic cells and suggested that "embryonic rests" were the source of tumors (Rather 1978). Later, the primitive cytoarchitecture and embryonic features of many malignant brain tumors was also described by Bailey and Cushing (1926). Smyth and Stern's (1938) observed that "subependymal glia may actually be the point of origin of tumors of the thalamus."

These earlier descriptions were further advanced in 1944, Joseph Globus and Hartwig Kuhlenbeck called attention to the subependymal cell plate in the adult brain and described this structure as one with primitive cellular composition. Based on a study with human brain tumors, they stated that "one of the most important sources for such immature embryonal residue from which neuroectodermal tumors are likely to develop under certain still unknown conditions is the subependymal plate." (Globus 1944).

The subependymal plate was better characterized as a "mitotically active and well defined subependymal layer is present in mammalian brains throughout life" and Lewis (1968) suggested that they could be a "possible source of different histological varieties of glioma, in particular those tumors in paraventricular situation and butterfly gliomas of the corpus callosum."

In the late 1960s Hopewell and Wright (1969) demonstrated increased glial tumors with periventricular implantation of carcinogens in rats. In the 1970s, periventricular tumors were then demonstrated to occur in the subventricular region after intraventricular inoculation with avian sarcoma viruses, with a much higher rate of tumors occurring in neonatal rats versus adult rats (Copeland et al 1975; Copeland and Bigner 1977; Vick et al 1977). A single dose of ethylnitrosurea administration to pregnant rats also induced periventricular tumors in the offspring (Koestner et al 1971).

Now clear evidence exists for the "subependymal plate, as described by Gobus and Kuhlenbeck is the subventricular zone (SVZ), know to be the largest cellular region of neural stem cells (NSCs) in the adult mammalian brain. The NSCs have been characterized by Buyalla and provide the migratory neuroblasts for neurogeneis 
in the olfactory bulb in rodents (Figure 1) (Sanai et al 2005). Recently, this migratory path for SVZ NSCs was also described in humans (Curtis et al 2007).

\section{Reappraising the prevailing theory of tumor genesis}

It has been widely accepted that cancer occurs as a consequence of genetic and epigenetic alterations in a differentiated cell. These alterations could provide a proliferative advantage and ultimately lead to uncontrolled growth and spread of the malignant cells. This theory suggests that tumors, such as gliomas, result from mutations to terminally differentiated astrocytes and oligodendrocytes that "de-differentiate" into a less differentiated phenotype (Mabon et al 1950; Doetsch et al 1999; Sakariassen et al 2007). Although the neoplastic transformation of fully differentiated glia is widely assumed to be the mechanism of gliomagenesis, this hypothesis has never been adequately tested.

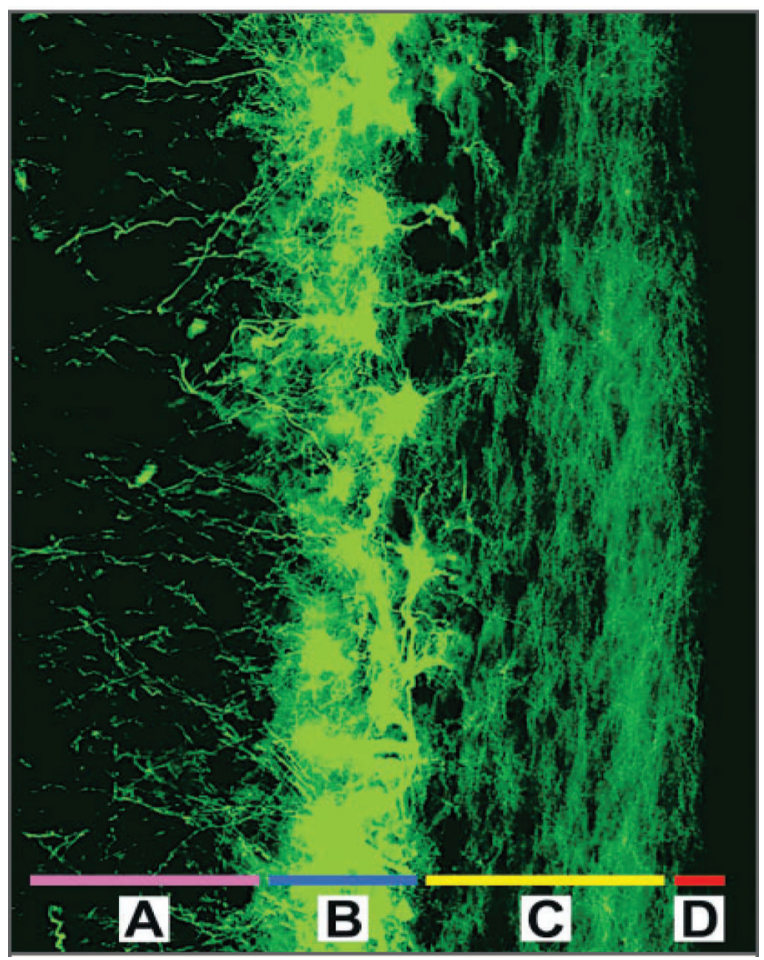

Figure I The cells of the subventricular zone, labeled with the astrocyte marker GFAP (shown in green), line the lateral walls of the lateral ventricles. This is the largest known region of adult neural stem cells in the human brain; it is composed of the deep subcortical white matter $\mathbf{A}$ ), a periventricular ribbon of astrocytes that can function as neural stem cells $\mathbf{B}$ ), a dense layer of astrocytic processes $\mathbf{C}$ ), and the ependymal lining D). Throughout adult life, astrocytes from the subventricular zone exhibit a unique capacity for multipotency and self-renewal in vitro. Copyright (C) 2005. Reproduced with permission from Sanai N,Alvarez-Buylla A, Berger M. 2005. Neural stem cells and the origin of gliomas. N Engl J Med, 353:8I I-22.

\section{Evidence for NSC as the cell of origin}

The neurogenic zones within the human central nervous system (CNS) with their resident NSCs are considered the leading candidates for transformation leading to brain tumors. Specifically mitotically active cells (NSCs and their direct progeny, transit amplifying cells) are the cells that have the greatest probability of being the brain tumor cell of origin.

\section{NSCs and gliomas share histological and biological similarities}

Gliomas have long been described by pathologists for their remarkable cellular heterogeneity, and in fact the most aggressive and malignant glioma (glioblastoma multiforme) was termed based partly on the histological diversity comprising this tumor, hence "multiform." A transformed NSC could provide this cellular landscape due to their mulitipotentiallity (ability to differentiate into the cell types that constitute their respective germ line). Mixed cell gliomas exist, such as oligoastrocytomas, and have both oligodendrocytes and astrocytes (Valtz et al 1991) and could be independent transformation of two differentiated cells, as suggested by the dedifferentiation theory of tumor genesis. More plausible would be the transformation of a single, bipotential progenitor cell such as a NSC or a transit amplifying cell (Chekenya and Pilkington 2002). These mixed cell gliomas also exhibit loss of heterozygosity on chromosomes $1 \mathrm{p}$ and $19 \mathrm{q}$ in both the astrocytic and oligodendrocytic components (Kraus et al 1995), suggesting that, in oligoastrocytomas, the astrocytes and oligodendrocytes comprising the tumor have a shared cell of origin.

Further, many glioma cells are undifferentiated, and lack expression of differentiated cell markers, as well as demonstrate staining with markers for nestin. Nestin expression is one hallmark feature of NSCs (Dahlstrand et al 1992; Tohyama et al 1992), and has become a reliable marker of NSCs (Lendahl et al 1990). Gliomas and NSCs also exhibit characteristic overlapping behavior (Table 1) (Sanai et al 2005), such as high motility, association with vasculature and white matter tracts (Shoshan et al 1999; Doetsch et al 2002; Palmer et al 2000).

\section{NSCs more likely to accumulate oncogenic mutations}

Accumulation of oncogenic genetic hits by cells is an infrequent stochastic event that most likely takes considerable time to result in transformation. NSCs, defined by their ability to self renew are both mitotically active and exist during the lifetime 
Table I Characteristics intrinsic to neural stem cells and gliomas. Copyright (C) 2005. Reproduced with permission from Sanai N, Alvarez-Buylla A, Berger M. 2005. Neural stem cells and the origin of gliomas. N Engl J Med, 353:8I I-22

High motility

Diversity of progeny

Robust proliferative potential

Association with blood vessels

Association with white-matter tracts

Immature expression profiles

Nestin expression

EGF-receptor expression

PTEN expression

Hedgehog pathway activity

Telomerase activity

Wnt pathway activity

Abbreviation: EGF, epidermal growth factor.

of the animal, allowing them to potentially accumulate the necessary multiple mutations for tumor formation. Accordingly, the cellular origin of gliomas would most likely occur from the proliferative zones in the mammalian CNS such as the SVZ contain at least two types of mitotically active cells: NSCs and transit amplifying cells (TACs) (Seri et al 2004). Although TACs exist only briefly and then differentiate, their total cellular compartment is significantly larger than NSCs and total number of global number of divisions during their relatively short lifespan are comparable to NSCs that exist throughout life yet are less mitotically active (Figure 2) (Vescovi et al 2006).

\section{Cancer stem cells}

Approximately 150 years ago, pathologists Rudolph Virchow and Julius Cohnheim suggested there were histological similarities between the developing fetus and certain cancers (such as teratocarcinomas) and that both tissues have the capacity to differentiate and proliferate. This "embryonal-rest hypothesis" is the historical version of today's cancer stem cell (CSC) hypothesis (Huntly and Gilliland 2005). As defined at the American Association for Cancer Research workshop on cancer stem cells: cancer stem cells are cells that (1) self renew and (2) re-supply the tumor with the various lineages of cells of which it is comprised. Self renewal can only be defined experimentally by the ability to recapitulate the generation of a continuously growing tumor or tumor initiation cell (Figure 3) (Clarke et al 2006; Lee and Herlyn 2007).

The original work establishing the CSC model was based on the hematopoietic system. Evidence for leukemia-CSCs was first reported in 1994 when Lapidot and colleagues
A

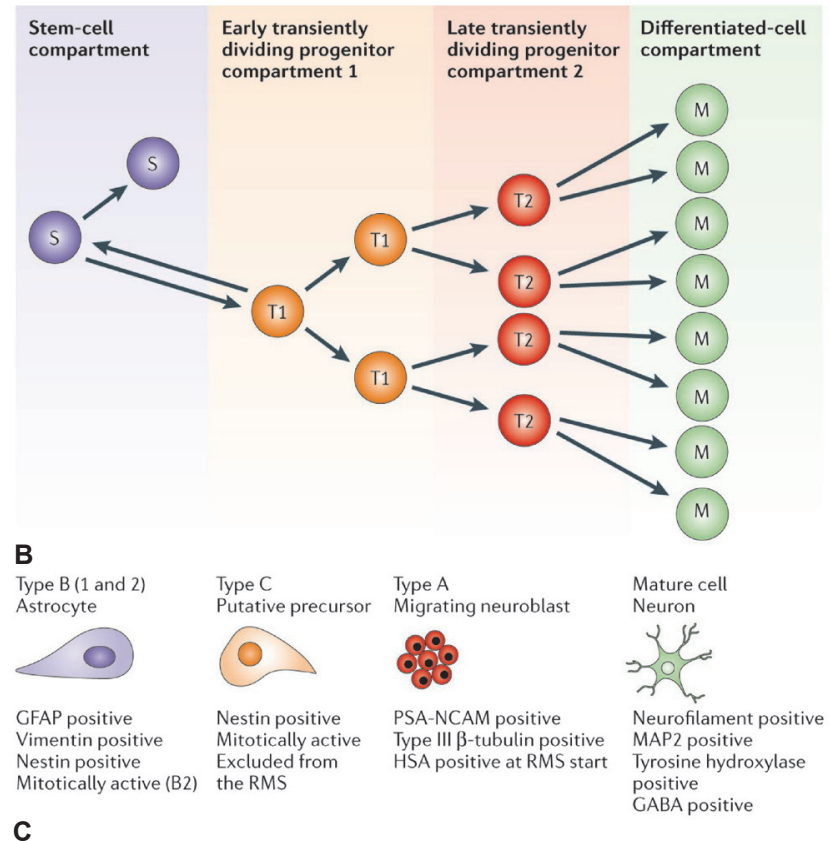

Adult subventricular zone

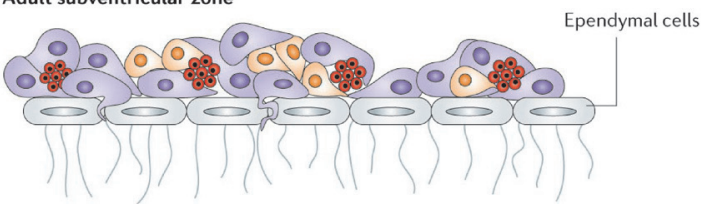

Figure 2 Hierarchical organization of the functional compartments in renewing tissues A) The stem-cell compartment (purple), early transiently dividing progenitor compartment (orange), late transiently dividing progenitor compartment (red) and differentiated-cell compartment (green) are schematically described. Cells in the stem-cell and transiently dividing progenitor compartments could be the target of the onco-transformation that leads to the formation of tumour stem cells B) The neural precursors that make up similar functional compartments in the neurogenetic regions of the adult brain and that might be the source of brain tumour stem cells C) The structure of the subventricular zone, showing how these precursors fit and are organized in the germinal neuroepithelium of the largest neurogenetic region of the adult brain.

Abbreviations: GABA, $\gamma$-aminobutyric acid; GFAP, glial fibrillary acid protein; HSA, heat-stable antigen; MAP2, microtubule-associated protein 2; NCAMI, neural cell adhesion molecule I; PSA, polysialic acid; RMS, rostral migratory stream.

isolated a rare population of CD34+CD38 - cells from patients with acute myeloid leukemia. Infusion of these CD34+CD38- cells into severe combined immune-deficient mice resulted in leukemic blast generation; however, more differentiated cells (CD34+ CD38+) did not generate leukemia (Lapidot et al 1994; Al-Hajj et al 2003; Buzzeo et al 2007). On a molecular level, CSCs share properties with normal stem cells. They have similar markers and signaling pathways, respond to environmental cues, as well as telomerase activity, apoptosis clearance and increased membrane transporter activity (Sakariassen et al 2007).

The first report of cells with stem-like properties in brain tumors was by Ignatova and colleagues (2002) where surgical specimens of glioblastoma multiforme were shown to have 


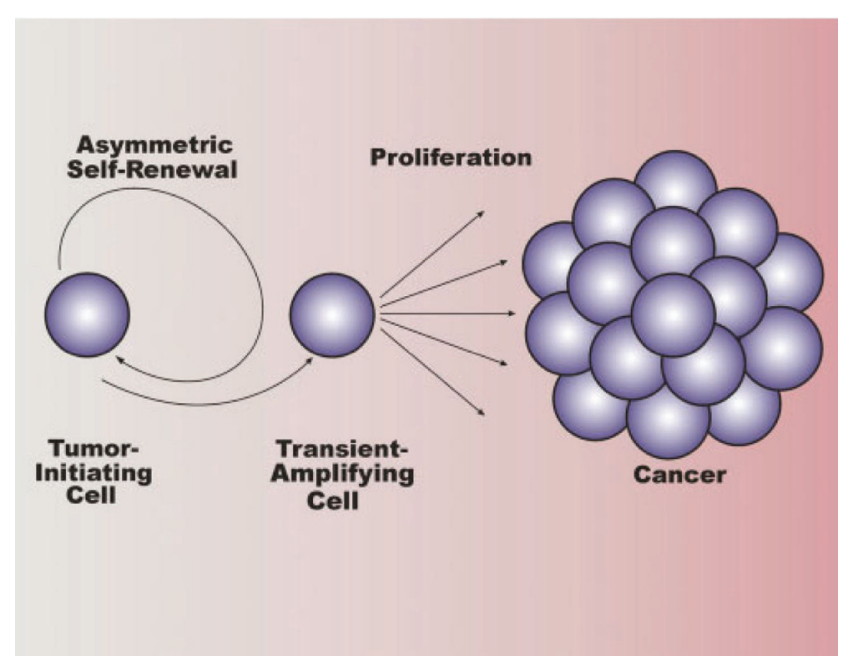

Figure 3 Overview of tumor stem cells in cancer. Cancer stem cells (tumor-initiating cells) divide asymmetrically, resulting in self-renewal of the tumor-initiating cell and production of a daughter cell known as a transient-amplifying cell (progenitor cell). The transient- amplifying cell is not thought to possess self-renewing capabilities, but instead divides indefinitely to contribute to cancer progression. Copyright @ 2007. Reproduced with permission from Lee J, Herlyn M. 2007. Old disease, new culprit: tumor stem cells in cancer.J Cell Physiol, 213:603-9.

clonogenic neurosphere-forming cells that expressed both neuronal and glial markers upon differentiation (Figure 4) (Ignatova et al 2002; Vescovi et al 2006). Subsequently, the Dirks group demonstrated CSC in brain tumors by 1 transplantation of CD133+ or CD133- populations into immunodeficient mice. With as few as $100 \mathrm{CD} 133+$ cells from the primary tumor, a new phenocopy of the tumor could be created in the transplanted mice; and unsorted or CD133- primary tumor cells were unable to cause de novo tumor generation. As part of what has come to define CSCs-self-renewal capacity - was also shown by confirming the ability of serially transplanted CD133+ cells to recapitulate the original tumor (Singh et al 2004; Buzzeo et al 2007). These findings established the presence of brain tumor stem cells (BTSCs), cells which can differentiate into the neural lineages, and exhibit self renewal as demonstrated by recapitulation of primary tumors with serial transplantation. This has been shown for other types of brain tumors as well (Merkle et al 2004; Taylor et al 2005). The existence of these BTSCs adds further evidence toward the NSCs origin of gliomas by confirming that different brain tumors contain transformed, undifferentiated neural precursors that respond to the same mitogens that activate adult NSCs (Vescovi et al 2006). Second, they indicate that tumor stem-like cells possess some of the molecular features of NSCs. Third, BTSCs, through asymmetric division, could generate a BTSCs and a progenitor cell, the latter of which may migrate away to either form or contribute to the tumor mass (Vescovi et al 2006, Berger et al 2004).

\section{Gliomas and NSCs have common regulatory pathways}

Neural stem cells and progenitor cells have activated cellular pathways, such as pro-mitotic genes, telomerase activity, and anti-apoptotic genes. This innate capacity overlaps with the mechanisms underlying tumor initiation, progression, or both. Thus, NSCs may require the least amount of mutations to become transformed.

1. EGFR expression is up-regulated in primary glioblastoma multiforme and transiently dividing progenitors (type $\mathrm{C}$ cells) (Mellinghoff et al 2005).

2. Fibroblast growth factors (FGFs) are involved in tumor proliferation and angiogenesis (Joy et al 1997; Auguste et al 2001) and also shown to regulate NSC proliferation and cell fate (Vescovi et al 1993; Gritti et al 1996; Palmer et al 1999).

3. Notch receptors and signaling is involved in NSC renewal (Hitoshi et al 2002; Shen et al 2004) and related to proliferative capacity of gliomas (Purow et al 2005).

4. PTEN is a tumor suppressor with an important function in the control of proliferation of neural stem cells and progenitor cells in vivo and in vitro (Baker and McKinnon 2004; Groszer et al 2001; Reya and Clevers 2005). PTEN is inactivated in glioblastomas (Rasheed et al 1999; Wechsler-Reya and Scott 2001) and its preservation in glioblastoma multiforme is clinically favorable (Mellinghoff et al 2005).

5. The Wnt-catenin pathway regulates adult neurogenesis (Chenn and Walsh 2003; Lie et al 2005) modulating its activity may increase glioma cell growth (Roth et al 2000).

Recent experiments have also highlighted the increased ability of progenitor cells to be transformed versus differentiated cell types. If epidermal growth factor receptor (EGFR) is transfected into transgenic Ink4a-Arf-/- mouse (lacking genes for cell-cycle arrest) neural stem cells, the cells lead to glioma formation (Bachoo et al 2002). This contrasted with similar manipulation of differentiated mouse astrocytes. Further, if the undifferentiated mouse astrocytes were transfected with platelet-derived growth factor ( $p d g f$ ) transgene and converted to a less differentiated state, they showed increased oncogenicity (Dai et al 2001; Bachoo et al 2002; Uhrbom et al 2002).

\section{Mouse models of gliomas for investigation of glioma origin}

Mouse models of gliomas are available and offer a unique opportunity to investigate tumor origin. These models, 

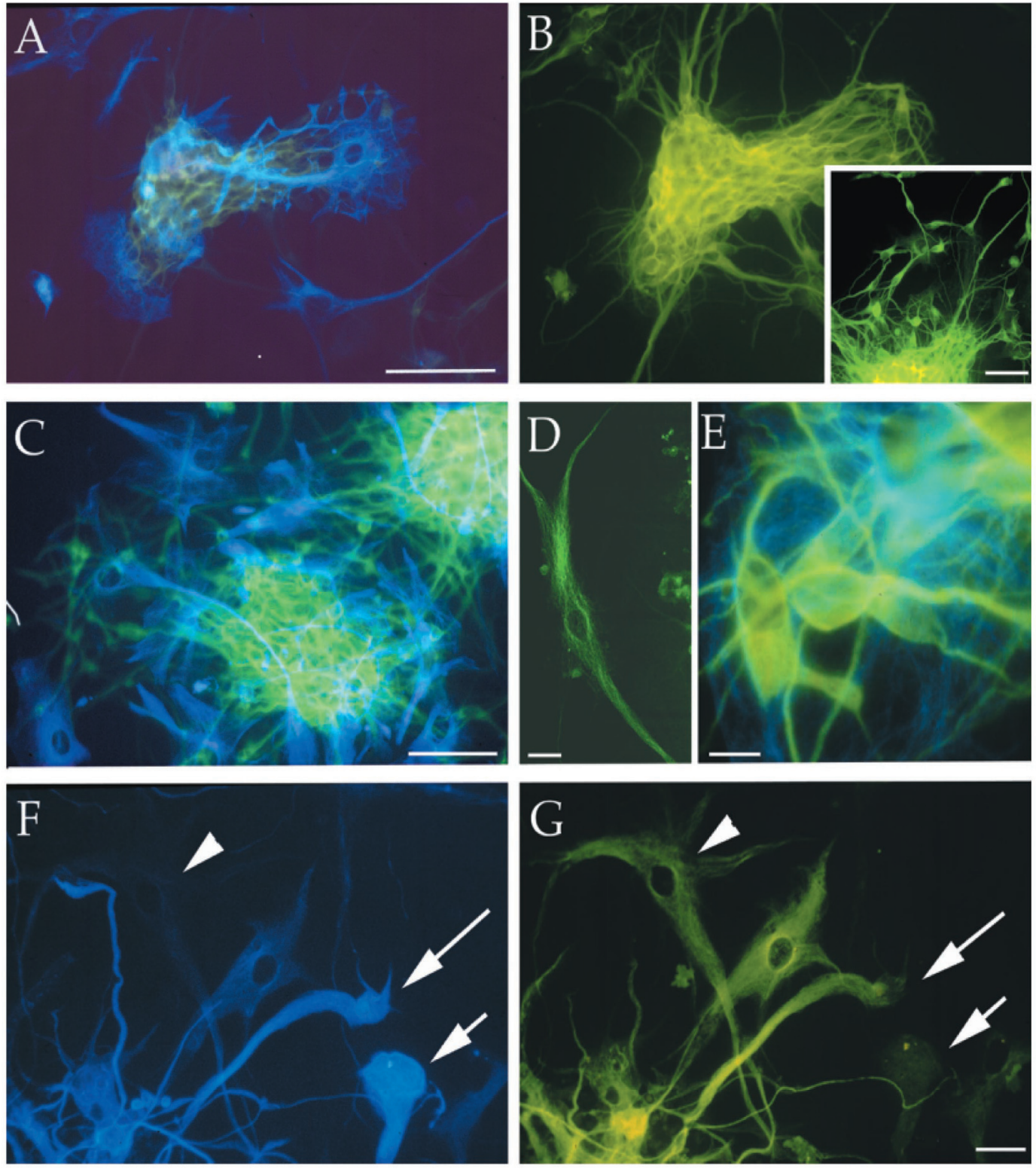

Figure 4 Immunofluorescence photomicrographs of representative MC-PL clones derived from different cortical glial tumor specimens (TI-T3, T6, and T7) processed for double (A, B, C, E, F) or single (B inset, D) labeling for different neural markers. The cells positive for the neuronal marker $\beta$-III tubulin are stained green (FITC), and the cells that expressed the astroglial marker GFAP are stained blue (AMCA). The arrowhead in F points out a cell that is not labeled for GFAP, but is immunopositive for $\beta$-III tubulin (arrowhead in $\mathbf{G}$ ). The short arrow in $\mathbf{F}$ points out a cell that is immunolabeled for GFAP, but immunonegative for $\beta$-III tubulin (short arrow in $\mathbf{G}$ ). The long arrow in $\mathbf{F}$ and $\mathbf{G}$ points out a cell that is labeled with both immunomarkers. Scale bars are $10 \mu \mathrm{m}$ (F and G) and I00 $\mu \mathrm{m}$ (A-E). Copyright @ 2002 . Ignatova T, Kukekov V, Laywell E, et al. 2002. Human cortical glial tumors contain neural stem-like cells expressing astroglial and neuronal markers in vitro. Glia, 39:193-206.

unlike glioma models in Drosophila and C. elegans, recapitulate the human pathology in terms of such characteristic structures as pleomorphic nuclei, diffusely infiltrative margins, secondary structures of Scherer, necrosis with pseudopalisading tumor cells, and microvascular proliferation. Xenograft models fail to phenocopy the classic histopathological features and are not an option for elucidating developmental mechanisms. Ultimately, these models of spontaneous tumor development and progression allows for the potential identification of novel mechanisms for tumorigenesis (Ding et al 2001).

\section{Relevance of identifying the glioma cell of origin}

Brain tumor classification with current histological criteria fails to accurately categorize patients as many patients with similar grade brain tumors have highly variable clinical outcomes. Clearly, this classification is one that at a minimum 
needs molecular modifiers. Defining the cell of origin, and confirming whether NSCs are indeed the cell of origin would improve not only glioma classifications, but also detection and treatment. The differential antigenic and molecular attributes of NSCs responsible for tumorigenesis could be exploited to target malignant cells prior tumor progression to clinical presentation. Indeed, defining cell of origin could help expand the concept of chemoprevention: targeting cells in the pre-morbid state.

\section{Disclosure}

The authors report no conflicts of interest in this work.

\section{References}

Al-Hajj M, Wicha M, Benito-Hernandez A, et al. 2003. Prospective identification of tumorigenic breast cancer cells. Proc Natl Acad Sci US A, 100:3983-8.

Auguste P, Gürsel D, Lemière S, et al. 2001. Inhibition of fibroblast growth factor/fibroblast growth factor receptor activity in glioma cells impedes tumor growth by both angiogenesis-dependent and independent mechanisms. Cancer Res, 61:1717-26.

Bachoo R, Maher E, Ligon K, et al. 2002. Epidermal growth factor receptor and Ink4a/Arf: convergent mechanisms governing terminal differentiation and transformation along the neural stem cell to astrocyte axis. Cancer Cell, 1:269-77.

Bailey P, Cushing H. 1926. A classification of the tumors of the glioma group on a histogenetic basis with a correlated study of prognosis. Philadelphia, PA: JB Lippincott Company.

Baker S, McKinnon P. 2004. Tumour-suppressor function in the nervous system. Nat Rev Cancer, 4:184-96.

Berger F, Gay E, Pelletier L, et al. 2004. Development of gliomas: potential role of asymmetrical cell division of neural stem cells. Lancet Oncol, 5:511-4.

Buzzeo M, Scott E, Cogle C. 2007. The hunt for cancer-initiating cells: a history stemming from leukemia. Leukemia, 21:1619-27.

Chekenya M, Pilkington G. 2002. NG2 precursor cells in neoplasia: functional, histogenesis and therapeutic implications for malignant brain tumours. J Neurocytol, 31:507-21.

Chenn A, Walsh C. 2003. Increased neuronal production, enlarged forebrains and cytoarchitectural distortions in beta-catenin overexpressing transgenic mice. Cereb Cortex, 13:599-606.

Clarke M, Dick J, Dirks P, et al. 2006. Cancer stem cells - perspectives on current status and future directions: AACR Workshop on cancer stem cells. Cancer Res, 66:9339-44.

Copeland D, Bigner D. 1977. The role of the subependymal plate in avian sarcoma virus brain tumor induction: comparison of incipient tumors in neonatal and adult rats. Acta Neuropathol (Berl), 38:1-6.

Copeland D, Vogel F, Bigner D. 1975. The induction of intractranial neoplasms by the inoculation of avian sarcoma virus in perinatal and adult rats. J Neuropathol Exp Neurol, 34:340-58.

Curtis M, Kam M, Nannmark U, et al. 2007. Human neuroblasts migrate to the olfactory bulb via a lateral ventricular extension. Science, 315:1243-9.

Dahlstrand J, Collins V, Lendahl U. 1992. Expression of the class VI intermediate filament nestin in human central nervous system tumors. Cancer Res, 52:5334-41.

Dai C, Celestino J, Okada Y, et al. 2001. PDGF autocrine stimulation dedifferentiates cultured astrocytes and induces oligodendrogliomas and oligoastrocytomas from neural progenitors and astrocytes in vivo. Genes Dev, 15:1913-25.

Ding H, Roncari L, Shannon P, et al. 2001. Astrocyte-specific expression of activated p21-ras results in malignant astrocytoma formation in a transgenic mouse model of human gliomas. Cancer Res, 61:3826-36.
Doetsch F, Caillé I, Lim D, et al. 1999. Subventricular zone astrocytes are neural stem cells in the adult mammalian brain. Cell, 97:703-16.

Doetsch F, Petreanu L, Caille I, et al. 2002. EGF converts transit-amplifying neurogenic precursors in the adult brain into multipotent stem cells. Neuron, 36:1021-34.

Globus JH, Kuhlenbeck H. 1944. The subependymal plate (matrix) and its relationship to brain tumors of the ependymal type. J Neuropathol Exp Neurol, 3:1-35.

Gritti A, Parati E, Cova L, et al. 1996. Multipotential stem cells from the adult mouse brain proliferate and self-renew in response to basic fibroblast growth factor. $J$ Neurosci, 16:1091-100.

Groszer M, Erickson R, Scripture-Adams D, et al. 2001. Negative regulation of neural stem/progenitor cell proliferation by the Pten tumor suppressor gene in vivo. Science, 294:2186-9.

Hitoshi S, Alexson T, Tropepe V, et al. 2002. Notch pathway molecules are essential for the maintenance, but not the generation, of mammalian neural stem cells. Genes Dev, 16:846-58.

Hopewell J, Wright E. 1969. The importance of implantation site in cerebral carcinogenesis in rats. Cancer Res, 29:1927-31.

Huntly B, Gilliland D. 2005. Leukaemia stem cells and the evolution of cancer-stem-cell research. Nat Rev Cancer, 5:311-21.

Ignatova T, Kukekov V, Laywell E, et al. 2002. Human cortical glial tumors contain neural stem-like cells expressing astroglial and neuronal markers in vitro. Glia, 39:193-206.

Joy A, Moffett J, Neary K, et al. 1997. Nuclear accumulation of FGF-2 is associated with proliferation of human astrocytes and glioma cells. Oncogene, 14:171-83.

Koestner A, Swenberg J, Wechsler W. 1971. Transplacental production with ethylnitrosourea of neoplasms of the nervous system in Sprague-Dawley rats. Am J Pathol, 63:37-56.

Kraus J, Koopmann J, Kaskel P, et al. 1995. Shared allelic losses on chromosomes $1 \mathrm{p}$ and $19 \mathrm{q}$ suggest a common origin of oligodendroglioma and oligoastrocytoma. J Neuropathol Exp Neurol, 54:91-5.

Lapidot T, Sirard C, Vormoor J, et al. 1994. A cell initiating human acute myeloid leukaemia after transplantation into SCID mice. Nature, 367:645-8.

Lee J, Herlyn M. 2007. Old disease, new culprit: tumor stem cells in cancer. J Cell Physiol, 213:603-9.

Lendahl U, Zimmerman L, McKay R. 1990. CNS stem cells express a new class of intermediate filament protein. Cell, 60:585-95.

Lewis P. 1968. Mitotic activity in the primate subependymal layer and the genesis of gliomas. Nature, 217:974-5.

Lie D, Colamarino S, Song H, et al. 2005. Wnt signalling regulates adult hippocampal neurogenesis. Nature, 437:1370-5.

Mabon R, Svien H, Adson A, et al.1950. Astrocytomas of the cerebellum. Arch Neurol Psychiatry, 64:74-88.

Mellinghoff I, Wang M, Vivanco I, et al. 2005. Molecular determinants of the response of glioblastomas to EGFR kinase inhibitors. $N$ Engl J Med, 353:2012-24.

Merkle F, Tramontin A, García-Verdugo J, et al. 2004. Radial glia give rise to adult neural stem cells in the subventricular zone. Proc Natl Acad Sci U S A, 101:17528-32.

Palmer T, Markakis E, Willhoite A, et al. 1999. Fibroblast growth factor-2 activates a latent neurogenic program in neural stem cells from diverse regions of the adult CNS. J Neurosci, 19:8487-97.

Palmer T, Willhoite A, Gage F. 2000. Vascular niche for adult hippocampal neurogenesis. J Comp Neurol, 425:479-94.

Purow B, Haque R, Noel M, et al. 2005. Expression of Notch-1 and its ligands, Delta-like-1 and Jagged-1, is critical for glioma cell survival and proliferation. Cancer Res, 65:2353-63.

Rasheed B, Wiltshire R, Bigner S, et al. 1999. Molecular pathogenesis of malignant gliomas. Curr Opin Oncol, 11:162-7.

Rather L. 1978. The Genesis of Cancer: A Study in the History of Ideas. Baltimore MD: Johns Hopkins University Press.

Reya T, Clevers H. 2005. Wnt signalling in stem cells and cancer. Nature, 434:843-50. 
Roth W, Wild-Bode C, Platten M, et al. 2000. Secreted Frizzled-related proteins inhibit motility and promote growth of human malignant glioma cells. Oncogene, 19:4210-20.

Sakariassen P, Immervoll H, Chekenya M. 2007. Cancer stem cells as mediators of treatment resistance in brain tumors: status and controversies. Neoplasia, 9:882-92.

Sanai N, Alvarez-Buylla A, Berger M. 2005. Neural stem cells and the origin of gliomas. $N$ Engl J Med, 353:811-22.

Seri B, García-Verdugo J, Collado-Morente L, et al. 2004. Cell types, lineage, and architecture of the germinal zone in the adult dentate gyrus. J Comp Neurol, 478:359-78.

Shen Q, Goderie S, Jin L, et al. 2004. Endothelial cells stimulate self-renewal and expand neurogenesis of neural stem cells. Science, 304:1338-40.

Shoshan Y, Nishiyama A, Chang A, et al. 1999. Expression of oligodendrocyte progenitor cell antigens by gliomas: implications for the histogenesis of brain tumors. Proc Natl Acad Sci U S A, 96:10361-6.

Singh S, Hawkins C, Clarke I, et al. 2004. Identification of human brain tumour initiating cells. Nature, 432:396-401.

Smyth G, Stern K. 1938. Tumors of the thalamus: a clinico-pathological study. Brain, 61:339-74
Taylor M, Poppleton H, Fuller C, et al. 2005. Radial glia cells are candidate stem cells of ependymoma. Cancer Cell, 8:323-35.

Tohyama T, Lee V, Rorke L, et al. 1992. Nestin expression in embryonic human neuroepithelium and in human neuroepithelial tumor cells. $L a b$ Invest, 66:303-13.

Uhrbom L, Dai C, Celestino J, et al. 2002. Ink4a-Arf loss cooperates with KRas activation in astrocytes and neural progenitors to generate glioblastomas of various morphologies depending on activated Akt. Cancer Res, 62:5551-8.

Valtz N, Hayes T, Norregaard T, et al. 1991. An embryonic origin for medulloblastoma. New Biol, 3:364-71.

Vescovi A, Galli R, Reynolds B. 2006. Brain tumour stem cells. Nat Rev Cancer, 6:425-36.

Vescovi A, Reynolds B, Fraser D, et al. 1993. bFGF regulates the proliferative fate of unipotent (neuronal) and bipotent (neuronal/astroglial) EGF-generated CNS progenitor cells. Neuron, 11:951-66.

Vick N, Lin M, Bigner D. 1977. The role of the subependymal plate in glial tumorigenesis. Acta Neuropathol (Berl), 40:63-71.

Wechsler-Reya R, Scott M. 2001. The developmental biology of brain tumors. Annu Rev Neurosci, 24:385-428. 
